OPEN ACCESS

Edited by:

Evgeny V. Mishin,

Air Force Research Laboratory,

United States

Reviewed by:

Arnaud Masson,

European Space Astronomy Centre

(ESAC), Spain

Gennady Milikh,

University of Maryland, United States

${ }^{*}$ Correspondence:

Andrii Voshchepynets

anddrii.voshchepynets@irf.se

Specialty section:

This article was submitted to

Space Physics,

a section of the journa

Frontiers in Astronomy and Space

Sciences

Received: 30 November 2018 Accepted: 11 March 2019

Published: 02 April 2019

Citation:

Voshchepynets A, Barabash S, Holmstrom M and Frahm RA (2019) Active Experiments Beyond the Earth: Plasma Effects of Sounding Radar Operations in the lonospheres of Venus, Mars, and the Jovian System. Front. Astron. Space Sci. 6:17. doi: 10.3389/fspas.2019.00017

\section{Active Experiments Beyond the Earth: Plasma Effects of Sounding Radar Operations in the lonospheres of Venus, Mars, and the Jovian System}

\author{
Andrii Voshchepynets ${ }^{1 *}$, Stas Barabash ${ }^{1}$, Mats Holmstrom ${ }^{1}$ and Rudy A. Frahm ${ }^{2}$ \\ ${ }^{1}$ Swedish Institute of Space Physics (IRF), Kiruna, Sweden, ${ }^{2}$ Southwest Research Institute, San Antonio, TX, United States
}

The operation of powerful satellite- and rocket-born sounding radars is often accompanied by a heating/acceleration of the local electrons and ions. Intense fluxes of sounder accelerated particles were detected in Earth's ionosphere when the frequency of the radar transmitter was close to one of the fundamental plasma resonances: harmonic of the electron-cyclotron frequency, plasma, or upper-hybrid frequencies. Recently it was found that running a sounder in the ionosphere of the non-magnetized Mars results in similar effects. Ion and electron sensors of the ASPERA-3 experiment (Analyzer of Space Plasma and Energetic neutral Atoms) onboard the Mars Express spacecraft discovered acceleration of the local ionospheric ions and electrons from thermal threshold energies to 100's of eV during the active sounding phase of the onboard sounder. ESA and NASA missions being studied or under development to Jupiter (JUICE- JUpiter ICy moon Explorer) in 2022, Europa Clipper in 2023 and to Venus (EnVision) in 2032 and ISRO Venus obiter in 2023 will also carry powerful sounding radars. The purpose of this study is to investigate what mechanisms can cause acceleration of the plasma particles during operations of the proposed sounding radars in the Jovian system and Venusian ionosphere. Using the results of the previous studies and characteristics of the proposed sounding radars onboard JUICE, Europa Clipper, EnVision, and ISRO Venus Obiter, we define the optimal conditions for observations of sounder accelerated particles, depending on the local conditions, such as plasma density, composition, and intensity of the magnetic field. The EnVision and ISRO Venus Obiter radar operations are expected to result in the most pronounced acceleration of ions and electrons, an effect that can be used to improve the local plasma diagnostics.

Keywords: active experiments in space, particle acceleration, ionospheric sounding, Moons of Jupiter, Mars, Venus

\section{INTRODUCTION}

Ionospheric sounding has been a standard tool for probing the ionosphere for many years. Principles of the sounding are based on the reflection of the radio waves from the ionized component of Earth's upper atmosphere (Appleton, 1927). Ionospheric sounders operate by transmitting a short pulse at a fixed frequency, and then detecting any echoes that are reflected. 
These echoes relate to the altitude where the plasma frequency is equal to the wave frequency. By measuring the time delay between the transmission of the pulse and the time that the echo is received, the range to the reflection point can be computed. By sequentially stepping the transmitter frequency after each transmit-receive cycle, the time delay, and hence the range to the reflection point, can be determined as a function of frequency. However, since radio waves return to the groundbased radar from only the portion of the ionosphere below the level of maximum electron density, no information about the ionosphere above this level can be retrieved. To perform topside sounding, ionosondes were fist mounted on rockets (Knecht et al., 1961) and later on satellites [Alouette (Lockwood, 1963), ISIS 1, 2 (McAfee, 1969)].

The situation with topside sounding (satellite- and rocketborne sounders) turned out to be less analogous to bottomside sounding (ground-based radars) than was expected. Longterm echoes were observed at frequencies of the fundamental plasma resonances: the electron Langmuir frequency $f_{p e}$, the upper hybrid resonance $f_{U H}$, the electron-cyclotron $f_{c e}$ and its harmonics $n f_{c e}(n=2,3, \ldots)$ (Calvert and Goe, 1963; Lockwood, 1963). The life-time of these resonances was one or two orders of magnitude longer than the duration of the sounding radio pulse itself. The long durations of the echoes were attributed to the low group velocity of the electrostatic waves that can be generated during the pulse (see Muldrew, 1972 as a review). Moreover, these waves can be reflected by the natural density (for $f_{p e}$ and $f_{U H}$, McAfee, 1968) or magnetic field (for $n H_{c e}$ ) gradients (Fejer and Calvert, 1964). As a result of both these factors, the waves travel a relatively short distance $\left(10^{2}-10^{4} \mathrm{~m}\right)$ form the sounder with group velocities of $10^{3}-10^{5} \mathrm{~m} / \mathrm{s}$ and return back, causing long standing echoes.

As it was first pointed out by Oya (1971), it is often the case that the voltage applied to the sounding antenna is comparable to the thermal energy of the electrons. This enables development of a turbulent layer in the vicinity of the sounder, and thus, different non-linear processes should be considered. The first consideration of the stimulated plasma instability and nonlinear phenomena in a framework of the weak turbulence approximation was done by Oya (1971) and further developed by Kiwamoto and Benson (1979) and Benson (1982). Initially, the goal of these studies was to explain the sequence of diffuse plasma resonances, intense echoes observed between harmonics of the electron-cyclotron frequency. The suggested explanation involved cyclotron heating of the plasma surrounding the satellite by the high-power transmitter pulse with subsequent development of the Haris instability (Oya, 1971) or non-linear Landau damping (Kiwamoto and Benson, 1979) that produces observed diffuse echoes.

The first observations that confirmed energization of the plasma by a top-side sounder were made by the Soviet Interkosmos 19 satellite. Galperin et al. (1981) reported observing bursts of superthermal electrons with a mean energy of about $100 \mathrm{eV}$ detected when the onboard high-power radio transmitter was transmitting signals with the tune frequency close to the local plasma frequency. Similar bursts of accelerated electrons and ions were observed also by soft-particle spectrometers (SPS,
Heikkila et al., 1970) onboard the Canadian satellites ISIS 1 and 2 (James, 1983, 1987). These observations began an intense study of the sounder accelerated particles (SAP) due to the importance of the subject in order to (1) understand the non-linear plasma processes near an active antenna and (2) develop new types of active experiments in space. Different models were proposed to explain SAP observations (see Shuiskaya et al., 1990 for a review). Despite great interest to the physics of SAP, current understanding of the matter is far from being complete, mainly due to the lack of observations.

With greater surprise, a similar phenomenon was recently found in the data collected by particle instruments onboard the Mars Express spacecraft (MEX, Voshchepynets et al., 2018). MEX is the first-ever extraterrestrial planetary mission equipped with both a powerful sounder (for subsurface and ionosphere sounding) and ion and electron sensors (of the ASPERA3 package, Barabash et al., 2006). The ASPERA- 3 plasma measurements cover a period of more than 14 years (more than one solar cycle) and contain multiple observations of SAP. This large set of observations makes it possible to establish statistically reliable dependencies of distribution functions of the accelerated particles on the sounder pulse characteristics (frequency, timing) and the local environmental conditions (plasma density, temperature, composition, magnetic field). The acceleration of electrons and ions by sounders results in the particle beams with known characteristics that can be used for diagnostic purposes. For instance, injection of artificially created beams of energetic electrons is a well-known technique for remote diagnostics of space plasma (Paschmann et al., 2001).

Studying SAP phenomenon is of critical importance to future active experiments in space, a field at the start of the rebirth. The ESA mission to Jupiter - JUICE (JUpiter ICy moon Explorer)to be launched in 2022 is equipped with both a powerful sounder for subsurface sounding and a comprehensive particle package measuring electrons and ions over a broad energy range. The purpose of this study is to investigate if the sounder onboard JUICE can cause acceleration of the plasma particles in the ionospheres of the large moons Ganymede and Callisto as well as in the Jovian magnetosphere. Other future missions equipped with powerful radars currently known to the authors are Europa Clipper and EnVision.

This paper is organized as follows: The results of observations of the SAP in the ionospheres of Earth and Mars are presented in section 2. Section 3 provides a brief description of the existing theories of the SAP generation. In section 4 we discuss the SAP phenomenon in the context of future space missions.

\section{OBSERVATIONS OF SOUNDER ACCELERATED PARTICLES}

Overall there were four satellite missions that studied SAP phenomenon in the Earth's ionosphere: International Satellites for Ionospheric Studies (ISIS 1,2, James, 1983, 1987), Interkosmos 19 (Ik-19, Galperin et al., 1981), and Cosmos 1809 (Shuiskaya et al., 1990). The satellites were operational on orbits between 500 and 3,000 km altitudes for different periods from 
TABLE 1 | Particle detectors used in SAP studies.

\begin{tabular}{|c|c|c|c|c|c|c|}
\hline Mission & Instrument & Measured particles & Energy range & Energy resolution & Field of view & Sampling time \\
\hline \multicolumn{7}{|c|}{ EARTH IONOSPHERE } \\
\hline \multirow[t]{2}{*}{ ISIS 1,2 } & SPS & Electrons & $5.5 \mathrm{eV}-13.15 \mathrm{keV}$ & $24.7 \%$ & $13^{\circ} \times 25^{\circ}$ & $11 \mathrm{~ms}$ \\
\hline & & lons & $5 \mathrm{eV}-14.68 \mathrm{keV}$ & $35.5 \%$ & $13^{\circ} \times 25^{\circ}$ & $11 \mathrm{~ms}$ \\
\hline IK 19 & SF-3 & Electrons & $10 \mathrm{eV}-15 \mathrm{keV}$ & $19 \%$ & $10^{\circ} \times 30^{\circ}$ & $20 \mathrm{~ms}$ \\
\hline \multirow[t]{2}{*}{ Cosmos 1809} & SF-3M & Electrons & $10 \mathrm{eV}-10 \mathrm{keV}$ & $21 \%$ & $20^{\circ} \times 190^{\circ}$ & $10 \mathrm{~ms}$ \\
\hline & & Ions & $10 \mathrm{eV}-10 \mathrm{keV}$ & $28 \%$ & $20^{\circ} \times 190^{\circ}$ & $40 \mathrm{~ms}$ \\
\hline \multicolumn{7}{|c|}{ MARS IONOSPHERE } \\
\hline \multirow[t]{2}{*}{ MEX } & ELS & Electrons & $1 \mathrm{eV}-20 \mathrm{keV}$ & $8 \%$ & $4^{\circ} \times 360^{\circ}$ & $28 \mathrm{~ms}$ \\
\hline & IMA & lons & $1 \mathrm{eV}-30 \mathrm{keV}$ & $7 \%$ & $90^{\circ} \times 360^{\circ}$ & $120 \mathrm{~ms}$ \\
\hline
\end{tabular}

TABLE 2 | Sounders used in SAP studies.

\begin{tabular}{|c|c|c|c|c|c|}
\hline Mission & Length & Frequency range & Pulse duration & Pulse repetition frequency & Power supply \\
\hline \multicolumn{6}{|c|}{ EARTH IONOSPHERE } \\
\hline ISIS 1 & $73 \mathrm{~m}$ & $0.1-20 \mathrm{MHz}$ & $86 \mu \mathrm{s}$ & $45 \mathrm{~Hz}$ & $400 W$ \\
\hline ISIS 2 & $73 \mathrm{~m}$ & $0.1-20 \mathrm{MHz}$ & $86 \mu \mathrm{s}$ & $30 \mathrm{~Hz}$ & $400 W$ \\
\hline IK 19 & $50 \mathrm{~m}$ & $0.3-15.95 \mathrm{MHz}$ & $133 \mu \mathrm{s}$ & $58.6 \mathrm{~Hz}$ & $140-300 \mathrm{~W}$ \\
\hline Cosmos 1809 & $50 \mathrm{~m}$ & $0.3-15.95 \mathrm{MHz}$ & $133 \mu \mathrm{s}$ & $58.6 \mathrm{~Hz}$ & $140-300 \mathrm{~W}$ \\
\hline \multicolumn{6}{|c|}{ MARS IONOSPHERE } \\
\hline MEX (MARSIS) & $40 \mathrm{~m}$ & $0.1-5.5 \mathrm{MHz}$ & $91.4 \mu s$ & $127 \mathrm{~Hz}$ & $60 \mathrm{~W}$ \\
\hline
\end{tabular}

1969 to 1990 . These unique missions were equipped with both powerful radars used for ionospheric sounding and particle detectors that enabled the discovery and studies of the SAP phenomenon. Characteristic of the onboard sounders and particle detectors can be found in Tables 1, 2. SAP electrons and ions have typical energy ranges up to $300-500 \mathrm{eV}$ and 100-200 eV, respectively. Electrons show very narrow angular distributions within a restricted pitch angle range around $90^{\circ}$. Ions showed weak pitch angle dependence. SAP are observed in bursts, a burst has a duration of $0.3-3 \mathrm{~ms}$ (Galperin et al., 1981). The most intensive electron fluxes were registered when the transmitter frequencies corresponded to one of the resonance frequencies of the surrounding plasma. Accelerated ions are typically detected when the transmitter frequency is near the local plasma frequency. James (1983), based on the measurements of the ISIS 1,2 satellites, showed that accelerated electrons are detected also when the sounding frequency is close to the electron gyro-frequency or its harmonics. Later, Shuiskaya et al. (1990) reported that the SAP electrons can be detected when the sounder operates in the range of the diffuse resonances (a broad area between $n f_{c e}$ when harmonics are below $\left.f_{U H}\right)$, and between the second and third harmonics of the local plasma frequency.

Very interesting results were obtained from the data collected by the sounding rocket OEDIPUS-C (James et al., 1999; Huang et al., 2001). In this experiment, two suits of instruments were accommodated in two separate platforms connected by an electrically conducting tether that was closely aligned with the Earth's magnetic field during the flight. The forward payload included a high-frequency radio transmitter HEX, which operated in a frequency range $25 \mathrm{kHz}-8 \mathrm{MHz}$. A synchronized wave receiver called REX was located on the aft payload platform to monitor the characteristics of waves emitted from HEX. Both platforms were equipped with electron detectors that provided measurements of the electron fluxes within the range $10 \mathrm{eV}$ to $20 \mathrm{keV}$. On the forward platform, the transmission at the electron $f_{c e}$ (and sometimes its harmonics), HEX caused electron acceleration throughout the duration of the rocket flight. The maximal energy of the electrons detected during the OEDIPUS$\mathrm{C}$ experiments was above $10 \mathrm{keV}$, that is much higher than the SAP energy recorded by any previous mission. The aft payload also detected accelerated electrons in the similar energy range. Simultaneously with the electron receiver on aft payload, strong emissions in a whistler-mode frequency range $(200-700 \mathrm{kHz}$ for this experiment) were detected. Unlike the forward payload, the aft payload observed the accelerated electrons with a short delay ( $\sim 300 \mu \mathrm{s})$ following the start of transmission and persisted for a short period after the transmitter was turned off.

There are two types of space borne radio sounders. Radars of the first type are used for ionospheric and sub-surface sounding. They are designed to produce distant radio echoes in order to study ionospheric density profiles below the spacecraft or to acquire data about sub-surface structure. Sounders onboard ISIS, Ik-19, and MEX belong to this type of sounders. The second type, known as a relaxation sounder, is a low-power sounder designed to stimulate local plasma resonances. Sounders of this type were flown on numerous magnetospheric satellites such as ISEE 1 (Harvey et al., 1979), GEOS 1 and 2 (Etcheto and Bloch, 1978), CLUSTER (Décréau et al., 2001), and extraterrestrial missions, such as Ulysses (Stone et al., 1992) and Cassini 


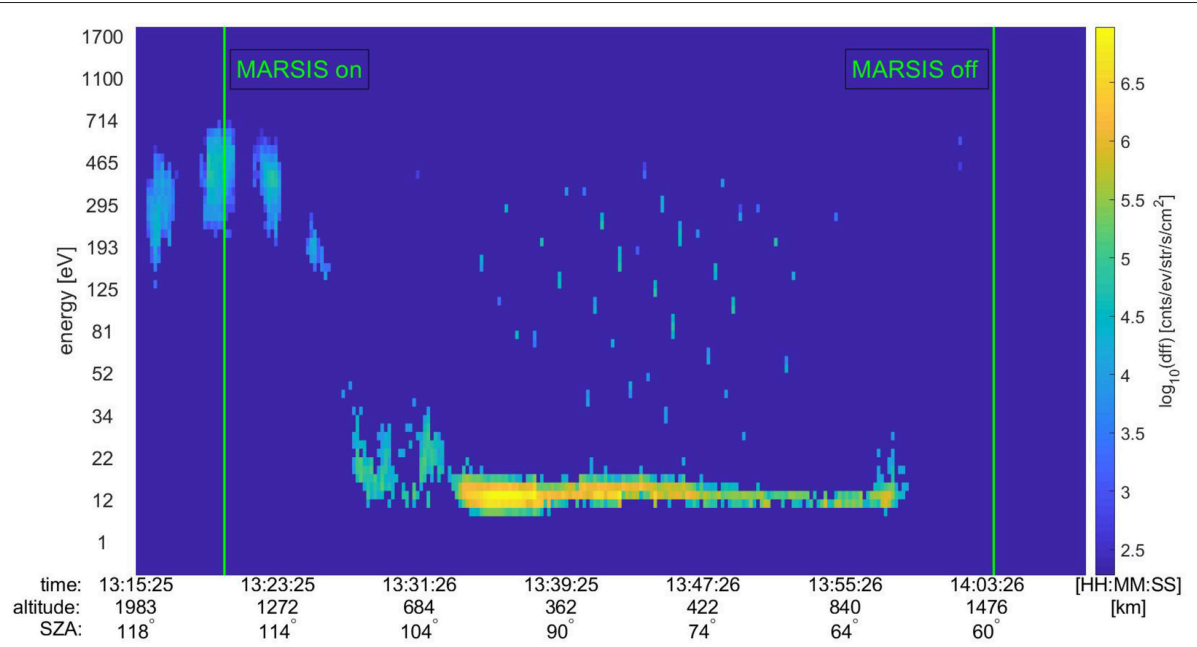

FIGURE 1 | Differential flux of $\mathrm{O}^{+}$ions measured by the ASPERA-3 IMA sensors on 22-03-2012 from 13:15 to 14:09 UTC.

(Gurnett et al., 2004). This type of sounders is optimized for measuring densities of very tenuous plasmas (down to $1 \mathrm{~cm}^{3}$ ). The working frequency of the relaxation sounders is thus much lower (typically below $100 \mathrm{kHz}$ ) than that of the ionospheric and sub-surface sounders. We found no publications on the SAP being detected on the missions equipped with the relaxation sounders. Some of the radars, for instance Radio Plasma Imager (Reinisch et al., 2000) onboard IMAGE satellite, can combine both types of sounding techniques, but IMAGE did not have any electron or ion sensors.

MEX is the first extraterrestrial mission equipped with both a powerful radar (MARSIS - Mars Advanced Radar for Subsurface and Ionosphere Sounding) (Jordan et al., 2009) and comprehensive particle instruments (ASPERA-3, Barabash et al., 2006). This combination of instrumentation enabled studies of the SAP phenomena under plasma conditions other than those in Earth's ionosphere. Characteristics of MARSIS and the ASPERA-3 ion (IMA - Ion Mass Analyzer) and electron (ELS ELectron Spectrometer) sensors are summarized in Tables 1, 2. Figure 1 shows an example of the sounder accelerated $\mathrm{O}^{+}$ions detected by IMA. The shown data were collected during $1 \mathrm{~h}$ around pericenter on orbit 10477. MARSIS started operating in ionospheric sounding mode at 13:20:13 UTC and stopped at 14:03:58 UTC. In this mode, the MARSIS operates in the frequency range $100 \mathrm{kHz} 5.5 \mathrm{MHz}$ sending $16091.7 \mu$ s pulses within $1.257 \mathrm{~s}$ and then remains idle for $6.285 \mathrm{~s}$. As one can see, a few minutes before crossing the terminator (around 13:39), IMA started detecting intense bursts of energetic ions with energies $40700 \mathrm{eV}$ on top of low energy $3-5 \mathrm{eV}$ ionospheric plasma background. The bursts were detected throughout the entire MARSIS operation period when the spacecraft was at altitudes below $800 \mathrm{~km}$. The time between two consecutive observations of the accelerated ions is found to be $15,23,38,60 \mathrm{~s}$ that coincides with 2, 3, 5, 8 MARSIS repetition times (7.54 s).

The SAP ions are routinely observed when MARSIS operated near pericenter (altitude $250 \mathrm{~km}$ ) on the day side of Mars.
Preliminary study of the data collected by MEX from 2007 to 2016 showed that 2,528 orbits (of 2,768 available) exhibit signatures of SAP ions. Observations of the accelerated electrons in the Martian ionosphere are much less frequent. Only several hundreds of orbits were found to exhibit signatures of SAP electrons. Figure 2 shows an example of sounder accelerated electrons detected by ELS together with SAP ions detected by IMA. In the Earth ionosphere, maximum energy of the SAP electrons is several times higher than that of ions, at Mars the situation is different. As one can see in Figure 2, the SAP ions are detected with energies higher than $400 \mathrm{eV}$, while energies of SAP electrons are below $200 \mathrm{eV}$. Analysis of a large number of observations gives similar results, the maximum energy of SAP ions is twice as high as the energy of SAP electrons (800 and $400 \mathrm{eV}$, respectively). Ions are detected when the frequency of the MARSIS pulse lies within the frequency range between local plasma frequency and its first harmonic. Electrons are typically detected when the MARSIS operating frequency matches the plasma frequency or one of the harmonics of the plasma frequency (between $2 f_{p e}$ and $5 f_{p e}$ ). The sampling times of IMA and ELS are 120 and $32 \mathrm{~ms}$ respectively. The sensors cannot resolve individual bursts of the SAPs lasting for $0.1 \mathrm{~ms}$ to a few milliseconds. The observed flux increases result from several bursts of SAPs that occur within one sampling period. A MARSIS pulse is $91.4 \mu$ s followed by the $7.9 \mathrm{~ms}$ sampling time. Therefore, during one IMA sampling time, there will be maximum 120/7.9 $=15$ SAP bursts per a IMA sampling time and 32/7.9 = 4 per ELS sampling time.

\section{THEORIES OF PARTICLE ACCELERATION BY A SOUNDER}

A number of explanations of particle acceleration by an active antenna in plasma have been proposed over the last 40 years (see Shuiskaya et al., 1990 and James et al., 1999 for a review). Due 

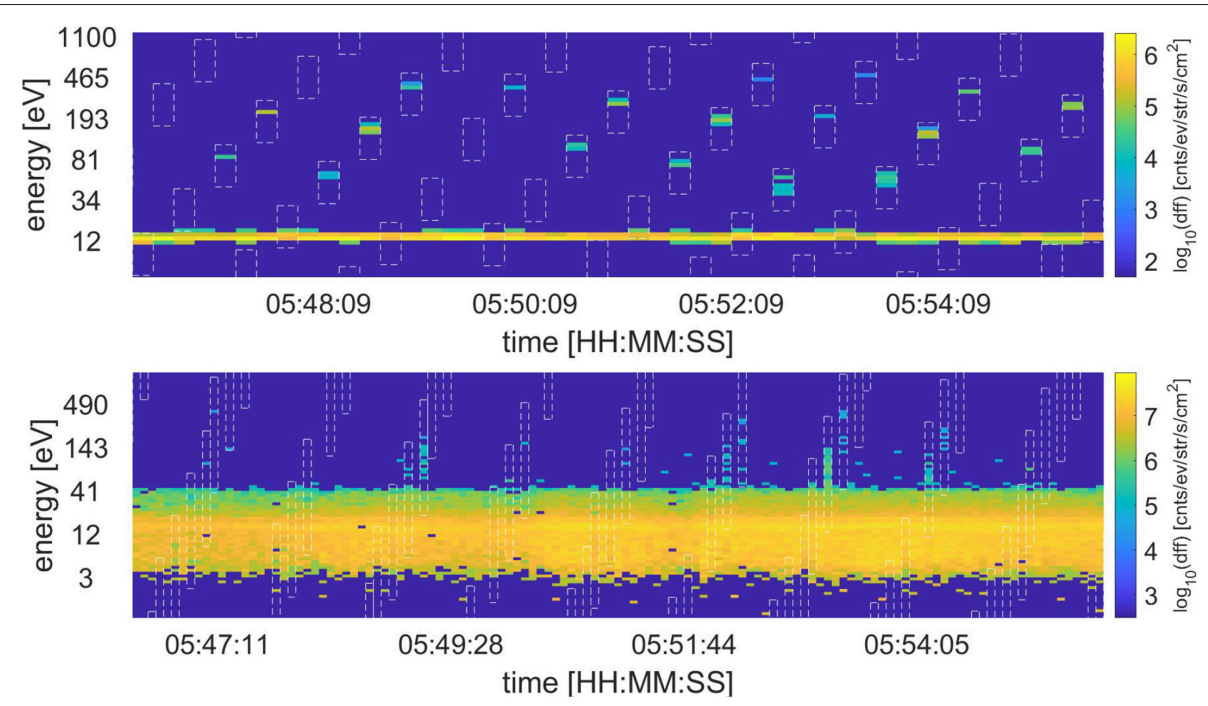

FIGURE 2 | The ASPERA-3 ions (Top) and electron (Bottom) measurements during MARSIS operations. White boxes indicate the $1.257 \mathrm{~s}$ time periods when MARSIS was scanning over frequencies. The fluxes of both accelerated ions and electrons correlate well with the periods when MARSIS was transmitting.
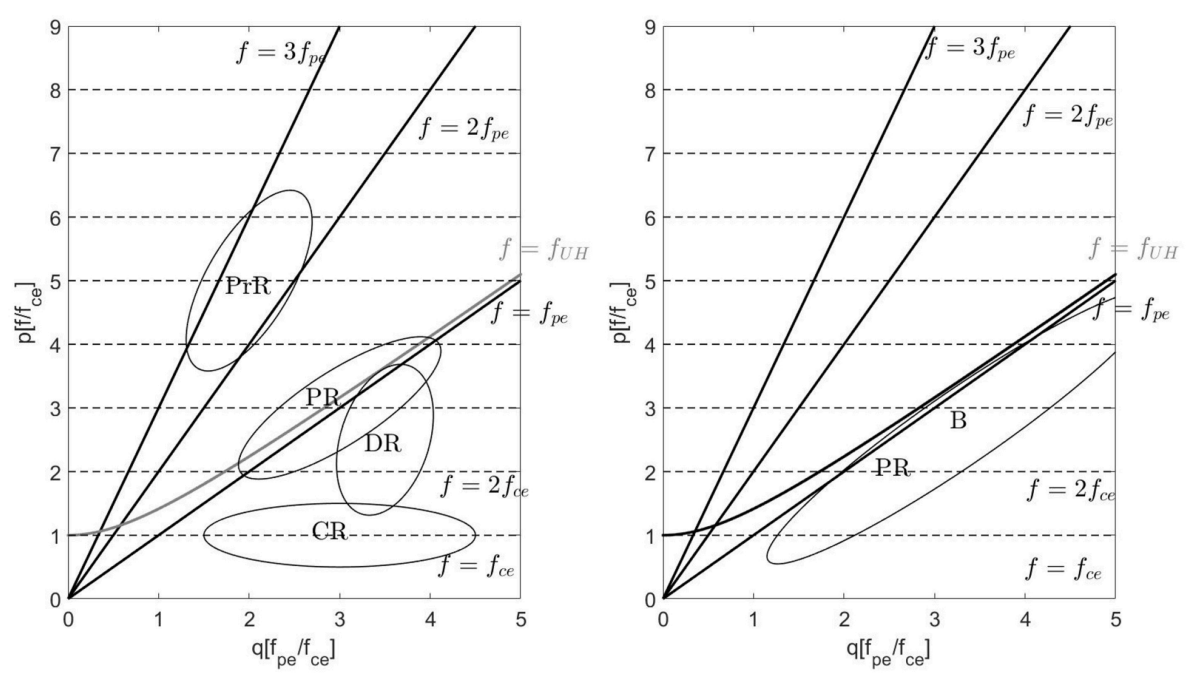

FIGURE 3 | Presentation of the SAP observations by Cosmos 1809 on the $p-q$ diagrams $\left(p=f / f_{c e}\right.$ and $\left.f_{p e} / f_{c e}\right)$. The selected areas show the locations of maximum electron (Left) and ion (Right) intensities together with the indication of the proposed acceleration mechanism. Electron acceleration models: cyclotron-resonance (CR) model (James, 1983), diffuse resonances (DR) model (Benson, 1982), plasma resonance (PR) model (Pulinets and Selegei, 1986), and parametric (PrR) resonance model (Serov et al., 1985). Ion acceleration models: plasma resonance (PR) model (Shuiskaya et al., 1990), wide-band acceleration (B) model (James, 1987). Local electron densities and magnetic fields are summarized in Table 3. Adapted from Shuiskaya et al. (1990).

to the fact that SAP are typically detected when the frequency of the transmitter is close to that of the fundamental plasma resonances, several models based on resonance wave-particle and wave-wave interaction have been suggested. Considering only acceleration of electrons, these are: cyclotron-resonance (CR) model (James, 1983), diffuse resonances (DR) model (Benson, 1982), plasma resonance (PR) model (Pulinets and Selegei, 1986), and parametric (PrR) resonance model (Serov et al., 1985). Cyclotron-resonance takes place when $f=f_{c e}$, where $f$ is the frequency of a transmitted pulse. In this model, electron heating occurs due to Landau damping of electromagnetic oscillations near the transmitting antenna. For the frequencies between $f_{c e}$ and $f_{p e}$, RF emission can generate electron-cyclotron waves near the antenna. These secondary waves are subject to strong non-linear Landau damping that also results in electron heating (DR model). The PR and PrR models consider electron acceleration in the framework of strong turbulence. In both models, the acceleration occurs as a result of a two-step process. The first stage involves development of the turbulent state near the antenna while the radar is transmitting and 
subsequent formation of the cavitons. This can be achieved by the modulation (at $f=f_{p e}$, PR model) or parametric (at $2 f_{p e}<$ $f<3 f_{p e}$, PrR model) instability. The collapse of the cavitons after the end of the transmission leads to a burst of Langmuir and ion-sound waves that can effectively accelerate electrons and ions due to the Landau damping. To classify SAP observations in a frequency domain, it is convenient to introduce the parameters $p=f / f_{c e}$ and $q=f_{p e} / f_{c e}$. In this case, each of the proposed mechanisms will occupy a certain area on the $p-q$ diagram, as can be seen in Figure 3 .

Another effect that is not included in the previously mentioned models is spacecraft charging resulting from the radar operations (James, 1983, 1987). A sinusoidal potential of the radar antenna with respect to the spacecraft during a pulse results in the currents to the spacecraft carrying by either ions or electrons, depending on the potential polarity. If the frequency of the transmitted pulse is lower than $f_{p e}$, the mobility of the ion and electrons is sufficient to restore the spacecraft potential to an equilibrium value after each half-wave of the pulse. If the frequency of the transmitted pulse is much higher than $f_{\text {pe }}$, neither electrons nor ions are sufficiently mobile to provide currents to change the spacecraft potential. If the frequency of the transmitted pulse is close to $f_{p e}$, the mobility of electrons is sufficient to charge the spacecraft but the plasma cannot support the sufficient ion current to restore the potential and the spacecraft becomes negatively charged to the amplitude of the pulse. The negatively charged spacecraft attracts and accelerates ions. This mechanism is often referred as wide-band acceleration and it is marked as B in the $p-q$ diagram.

Observations of SAP by MEX on the $p-q$ diagram are shown in Figure 4. For the present study, 30 orbits were selected that are characterized by the similar ionospheric environment conditions. Observations of SAP ions correspond to the region of PR and B mechanisms. Detection of the accelerated ions by IMA is often accompanied by a small decrease in low energy [10-100 eV] electron fluxes as one expects for a negatively charged spacecraft. The induced spacecraft charging model also explains the upper energy limit of SAP ions detected in the Martian ionosphere. As explained earlier, the model suggests that a steady negative DC potential on the order of the voltage applied to the antenna (400 $\mathrm{V}$ for MARSIS) is built up on the spacecraft when the transmitter is on. This implies that the instantaneous potential of the antenna with respect to the plasma/spacecraft oscillates from 0 to $-800 \mathrm{~V}$ resulting in the ion acceleration up to $800 \mathrm{eV}$ in agreement with observations. Observations of SAP electrons correspond to the $\mathrm{PR}$ and $\mathrm{PrR}$ regions on the $p-q$ diagram. It would indicate that the acceleration mechanism could be the resonant wave-particle interaction, but the issue is still under debate. Radar onboard MEX is much less powerful than the radars onboard Interkosmos 19 and ISIS-1,2 spacecrafts (60 W on MEX and $400 \mathrm{~W}$ on ISIS-1,2 and $300 \mathrm{~W}$ on Interkosmos 19). The case may be that the energy density of the radio waves transmitted by MARSIS is not high enough for the strong turbulence to develop.

\section{APPLICATION FOR THE FUTURE MISSIONS}

The JUpiter ICy Moons Explorer (JUICE) is an European Space Agency mission that will fly by and observe the Galilean satellites Europa, Ganymede, and Callisto, characterize the Jovian system in a lengthy Jupiter-orbit phase, and ultimately orbit Ganymede for in-depth studies of habitability, evolution, and the local environment (Grasset et al., 2013). It will be equipped with a powerful radar RIME (Radar for Icy Moons Exploration) (Bruzzone et al., 2013). RIME is optimized for the penetration

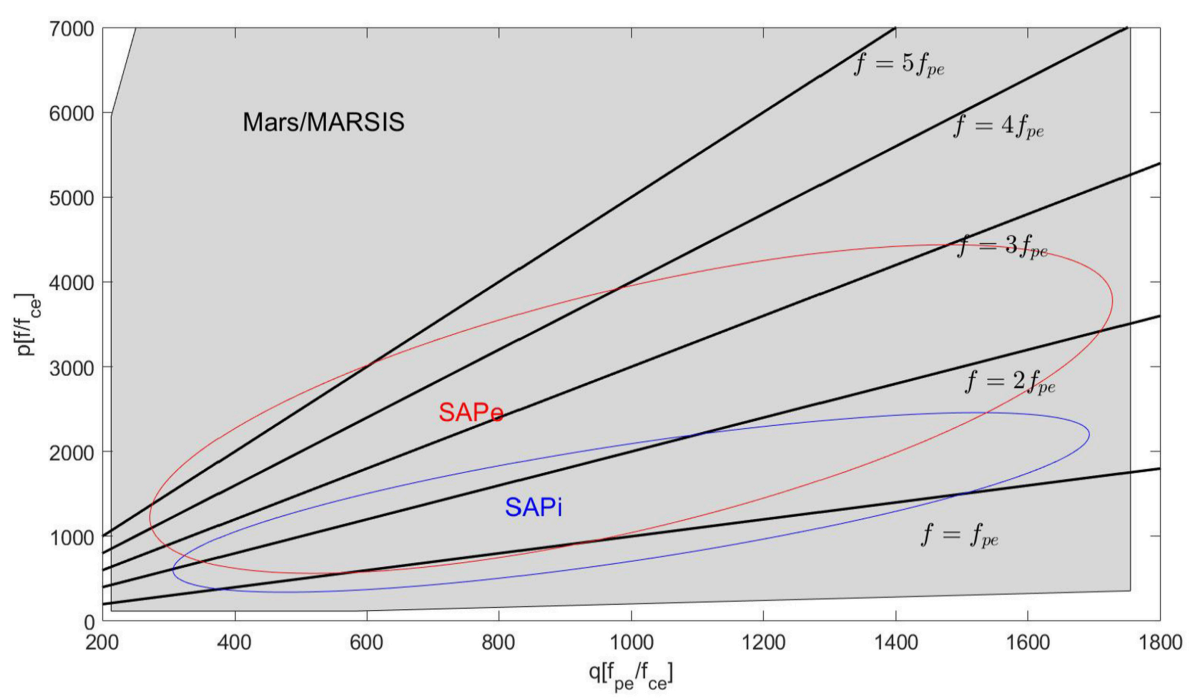

FIGURE 4 | Presentation of SAP observations by MEX on the $p-q$ diagram. The gray area shows the range of the $p-q$ parameters obtained for typical conditions of the Martian ionosphere (summarized in Table 3) and MARSIS sounding characteristics (summarized in Table 2). The areas encircled by blue and red lines indicate regions where SAP ions and electrons were detected. 
TABLE 3 | Local plasma characteristics (density and magnetic field) typical for SAP observations in the ionospheres of Earth and Mars.

\begin{tabular}{|c|c|c|c|c|}
\hline Target & Altitudes & Magnetic field & Electron density & References \\
\hline \multicolumn{5}{|l|}{ IK-19 } \\
\hline Earth & $500-1000$ km & $10-40 \mu \top$ & $10^{4}-10^{5} \mathrm{~cm}^{3}$ & Shuiskaya et al., 1990 \\
\hline Mars & $300-1000$ km & $10-30 \mathrm{nT}$ & $400-3000 \mathrm{~cm}^{3}$ & Fränz et al., 2006 \\
\hline \multicolumn{5}{|l|}{ JUICE } \\
\hline Callisto & $400-5000 \mathrm{~km}$ & $1-10 \mathrm{nT}$ & $10-400 \mathrm{~cm}^{3}$ & Gurnett et al., 2000; Russell, 2005 \\
\hline \multicolumn{5}{|c|}{ EUROPA CLIPPER } \\
\hline Europa & $25-5000 \mathrm{~km}$ & $100-200 \mathrm{nT}$ & $10^{2}-10^{4} \mathrm{~cm}^{3}$ & Kliore et al., 1997; Russell, 2005 \\
\hline Europa (plume) & $25-5000 \mathrm{~km}$ & $600-800 \mathrm{nT}$ & $500-5000 \mathrm{~cm}^{3}$ & Jia et al., 2018 \\
\hline \multicolumn{5}{|l|}{ ENVISION } \\
\hline
\end{tabular}

Anticipated range of plasma characteristic near Ganymede, Europa, Callisto and Venus for the JUICE, Europa Clipper, EnVision, and ISRO Venus Orbiter missions.

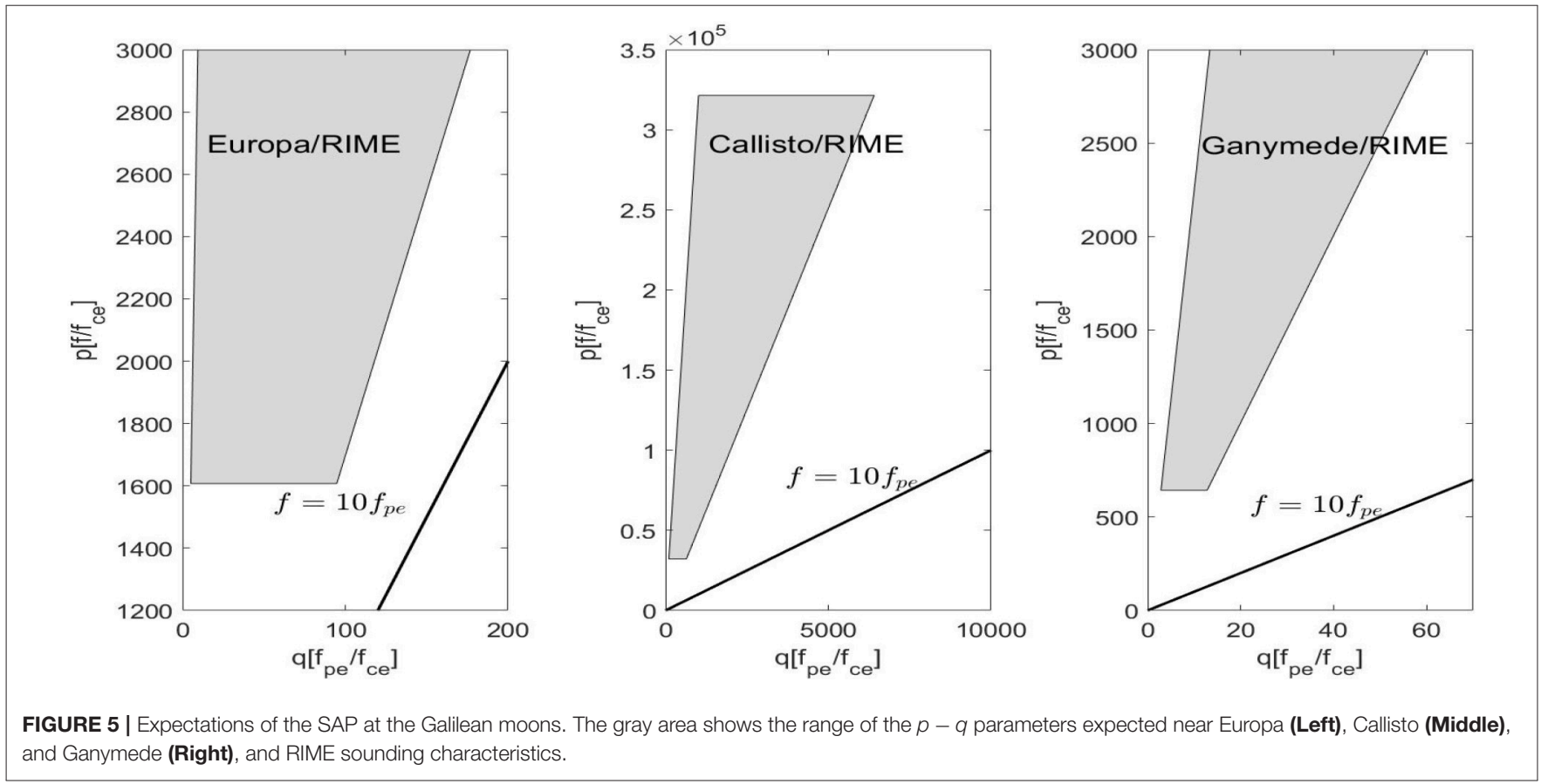

of the Ganymede, Europa and Callisto surfaces up to a depth of $9 \mathrm{~km}$ in order to allow the study of the subsurface geology and geophysics of the icy moons (in the search for possible subsurface water). In comparison to ionospheric sounders, radars used for subsurface studies transmit in a higher frequency range. For the RIME, the operating frequency will be set to $9 \mathrm{MHz}$. Analysis of the plasma conditions near Ganymede, Europa, and Callisto (Table 3) showed that RIME will operate far from any of the plasma resonances. Representation of the RIME characteristics with respect to the local plasma on the $q-p$ diagram is shown in Figure 5. It is highly unlikely that any mechanisms discussed in the previous section can produce SAP under the conditions expected at the Galilean moons - the RIME frequency is simply too high.

The similar situation is expected for the NASA reconnaissance mission Europa Clipper (Phillips and Pappalardo, 2014). The radar REASON (Radar for Europa Assessment and Sounding: Ocean to Near-surface, ) onboard Europa Clipper will have similar characteristics to the RIME and will operate at $9 \mathrm{MHz}$ (Schroeder et al., 2016). Despite the fact that the spacecraft will fly by Europe at much lower altitudes $(25 \mathrm{~km})$, the local plasma frequency will still be far below $9 \mathrm{MHz}$ (Figure 6). 
This can also be said about the plasma region affected by the water plume rising $\sim 200 \mathrm{~km}$ above Europa's surface (Roth et al., 2014). The local electron density should be as high as $10^{5} \mathrm{~cm}^{-3}$ in order to make it possible for SAP to occur.

The most promising missions for SAP studies beyond Earth orbit are EnVision, an orbital mission to Venus proposed to ESA (ESA proposal, Ghail et al., 2018) and ISRO Venus Orbiter proposed by the Indian Space Research Organization (Haider et al., 2018). EnVision will be placed on a circular low altitude orbit $(259 \mathrm{~km})$ and will carry a radar designed for subsurface studies (SRS). The radar will work with a central frequency in the range $9-30 \mathrm{MHz}$ for optimal ground penetration capability. Unlike Mars, Venus has a much denser atmosphere. Electron density in the

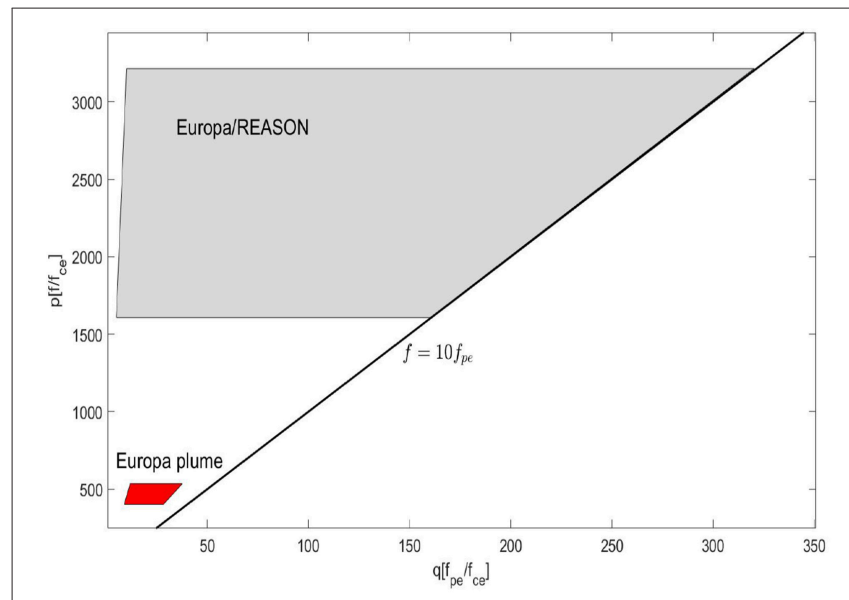

FIGURE 6 | Expectations of the SAP near Europa for the REASON radar. lower layers of the ionosphere of Venus can reach $10^{4}-$ $10^{5} \mathrm{~cm}^{-3}$ (Donahue and Russell, 1997). SRS onboard EnVision will thus operate in a range of $p-q$ parameters that corresponds to the range where MEX detected electrons and ions accelerated by MARSIS (Figure 7). ISRO Venus Orbiter will carry a sounding radar that should have characteristics similar to those of MARSIS on Mars Express. In the ionospheric sounding mode, the radar will be operating in a frequency range between 0.1 and $10 \mathrm{Mhz}$. This frequency range is well suited for SAP generation (Figure 8) near the periapsis $(500 \mathrm{~km})$ of the expected ISRO Venus Orbiter orbit. In addition to ion and electron sensors, ISRO Venus Orbiter will carry a plasma wave experiment, Venus Ionospheric Plasma wave detectoR (VIPER), that will provide measurements of electric and magnetic fields in the vicinity of the spacecraft. The combination of the sounder and particle instruments onboard ISRO Venus Orbiter will enable comprehensive study of the particle acceleration in the vicinity of the active antenna.

\section{CONCLUSION}

Particle acceleration by sounders in the planetary ionospheres is a type of active experiments of great interest. These phenomena are a result of routine operation of satellite-borne sounders and do not require any additional spacecraft or mission resources, an important factor for planetary missions with scarce resources. On the other hand, it provides additional opportunities for plasma diagnostics (see Voshchepynets et al., 2018). Nominal operations of the radars on the coming missions to Jupiter, JUICE and Europa Clipper, may not result in any notable SAP for typical plasma conditions due to too high radar frequency. However, the comprehensive plasma instrument

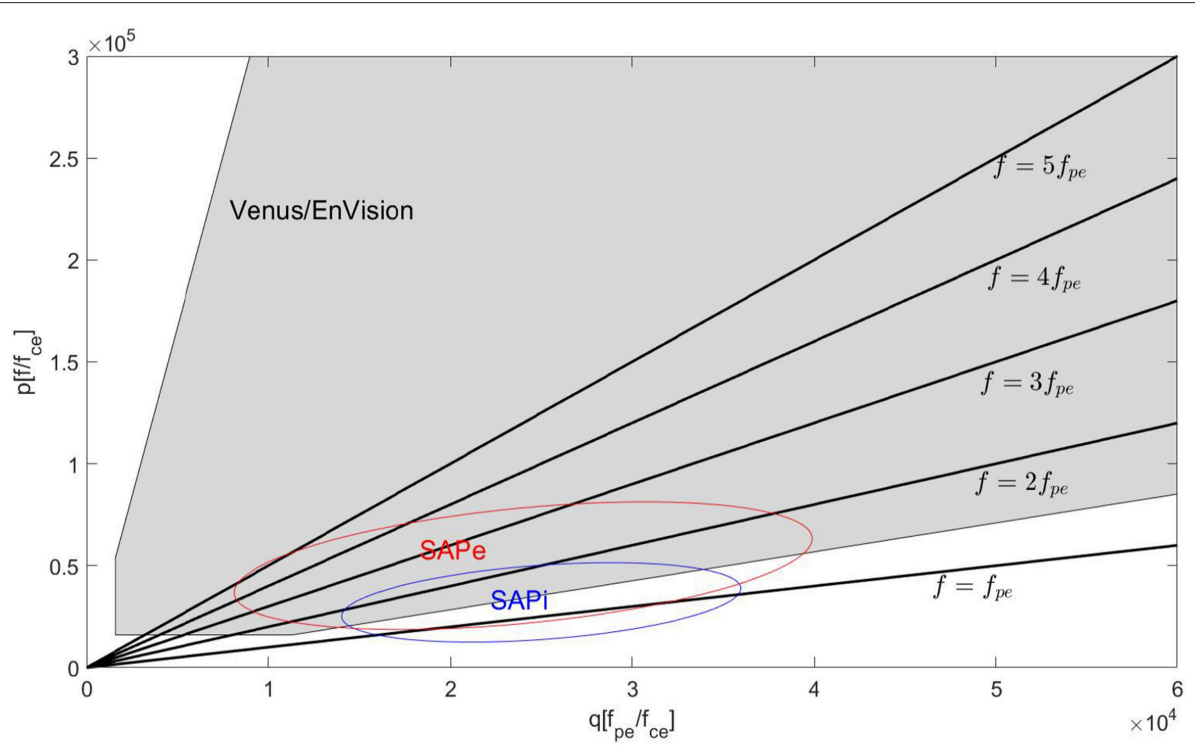

FIGURE 7 | Expectations of the SAP in the ionosphere of Venus. The gray area shows the range of the $p-q$ parameters for the expected Venus environment and SRS sounding characteristics. The areas encircled by red and blue indicate the possibility of the SAP detection. 


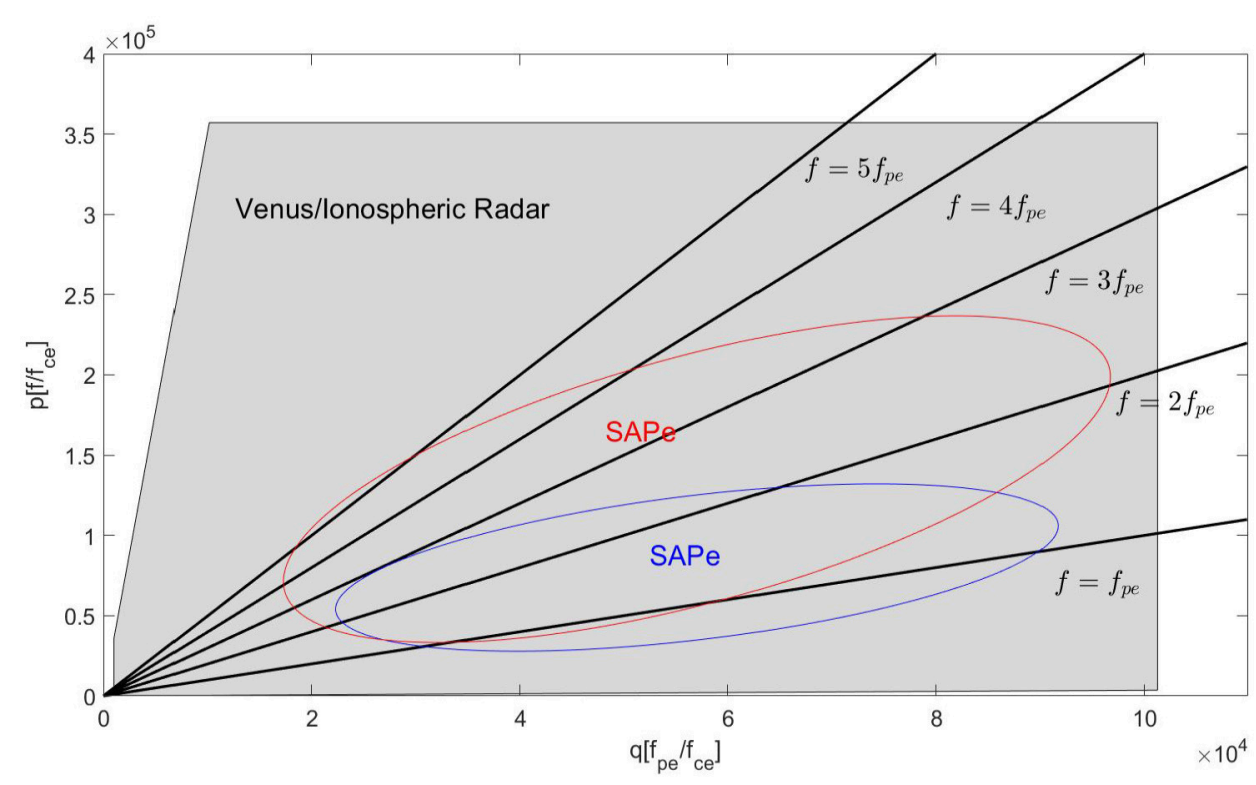

FIGURE 8 | Expectations of the SAP in the ionosphere of Venus. The gray area shows the range of the $p-q$ parameters for the expected Venus environment and SRS sounding characteristics of the radar onboard ISRO Venus Orbiter. The areas encircled by red and blue indicate the possibility of the SAP detection.

PEP (Particle Environment Package, Barabash et al., 2013) onboard JUICE could study plasma modification by an active antenna in the frequency domain far from the main plasma resonances $\left(f \gg f_{\text {pe }}, f_{c e}\right)$.

The sounders on the EnVision and ISRO Venus Orbiter missions to Venus are expected to produce strong SAP fluxes. Currently, EnVision is not equipped with a plasma instrument, but if an conventional ion and/or electron sensor will be added to the payload, they can fully utilize the plasma diagnostic technique made possible by SAP.

Another important aspect of the SAP phenomena is the conclusion on the high negative potential of the spacecraft body. While it occurs for a relatively short time of the order of few ms, it may have effect on the spacecraft platform or operations.

\section{DATA AVAILABILITY}

Data used in this study is available on the NASA Planetary Data System and the ESA Planetary System Archive.

\section{REFERENCES}

Appleton, E. V. (1927). The existence of more than one ionised layer in the upper atmosphere. Nature 120: 330. doi: 10.1038/120330a0

Barabash, S., Lundin, R., Andersson, H., Brinkfeldt, K., Grigoriev, A., Gunell, H., et al. (2006). The analyzer of space plasmas and energetic atoms (ASPERA-3) for the Mars express mission. Space Sci. Rev. 126, 113-164. doi: 10.1007/s11214-006-9124-8

Barabash, S., Wurz, P., Brandt, P., Wieser, M., Holmström, M., Futaana, Y., et al. (2013). "Particle Environment Package (PEP)," in European Planetary Science Congress (London) 8, EPSC2013-709

\section{AUTHOR CONTRIBUTIONS}

SB and AV contributed conception and design of the study. AV organized the database and performed the statistical analysis. All authors contributed to manuscript revision, read, and approved the submitted version.

\section{FUNDING}

We wish to acknowledge support through the Swedish National Space Agency to IRF in Sweden (Contract 169/15). At SwRI, work was conducted under NASA contract NASW-00003.

\section{ACKNOWLEDGMENTS}

The ASPERA-3 experiment on the ESA MEX mission is a joint effort of 15 laboratories in 10 countries, all sponsored by their national agencies. We thank all of these agencies as well as the various departments/institutions hosting these efforts.

Benson, R. F. (1982). Stimulated plasma instability and nonlinear phenomena in the ionosphere. Radio Sci. 17, 1637-1659. doi: 10.1029/ RS017i006p01637

Bruzzone, L., Plaut, J. J., Alberti, G., Blankenship, D. D., Bovolo, F., Campbell, B. A., et al. (2013). "RIME: radar for Icy Moon Exploration," in European Planetary Science Congress (London) 8, EPSC2013-744

Calvert, W., and Goe, G. B. (1963). Plasma resonances in the upper ionosphere. J. Geophys. Res. 68:6113. doi: 10.1029/JZ068i022p06113

Cravens, T. E., Shinagawa, H., and Luhmann, J. G. (1997). "Magnetohydrodynamic processes: magnetic fields in the ionosphere of venus," in Venus II: Geology, Geophysics, Atmosphere, and Solar Wind 
Environment, eds. S. W. Bougher, D. M. Hunten, and R. J. Phillips (Tucson, AZ: University of Arizona Press), 61.

Décréau, P. M. E., Fergeau, P., Krasnoselskikh, V., Le Guirriec, E., Lévêque, M., Martin, P., et al. (2001). Early results from the Whisper instrument on Cluster: an overview. Annal. Geophys. 19, 1241-1258. doi: 10.5194/angeo-19-1241-2001

Donahue, T. M., and Russell, C. T. (1997). "The venus atmosphere and ionosphere and their interaction with the solar wind: an overview," in Venus II: Geology, Geophysics, Atmosphere, and Solar Wind Environment, eds S. W. Bougher, D. M. Hunten, and R. J. Phillips (Tucson, AZ: University of Arizona Press), 3.

Etcheto, J., and Bloch, J. J. (1978). Plasma density measurements from the GEOS-1 relaxation sounder. Space Sci. Rev. 22, 597-610. doi: 10.1007/BF00223943

Eviatar, A., Vasyliūnas, V. M., and Gurnett, D. A. (2001). The ionosphere of Ganymede. Planet. Space Sci. 49, 327-336. doi: 10.1016/S0032-0633(00)00154-9

Fejer, J. A., and Calvert, W. (1964). Resonance effects of electrostatic oscillations in the ionosphere. J. Geophys. Res. 69, 5049-5062. doi: 10.1029/JZ069i023p05049

Fränz, M., Dubinin, E., Roussos, E., Woch, J., Winningham, J. D., Frahm, R., et al. (2006). Plasma moments in the environment of mars. Mars express ASPERA-3 observations. Space Sci. Rev. 126, 165-207. doi: 10.1007/s11214-006-9115-9

Galperin, I. I., Sagdeev, R. Z., Shuiskaia, F. K., Lisakov, I. V., Migulin, V. V., Kushnerevskii, I. V., et al. (1981). Detection of electron acceleration in the ionospheric plasma under the influence of high-power radio radiation near the local plasma frequency aboard the space vehicle Interkosmos 19. Cosmic Res. $19,34-44$.

Ghail, R., Hall, C., Mason, T., Herrick, L., Carter, C., Williams, J., et al. (2018). VenSAR on EnVision: taking earth observation radar to Venus. Int. J. Appl. Earth Observ. Geoinform. 64, 365-376. doi: 10.1016/j.jag.2017.02.008

Grasset, O., Dougherty, M. K., Coustenis, A., Bunce, E. J., Erd, C., Titov, D., et al. (2013). JUpiter ICy moons Explorer (JUICE): an ESA mission to orbit Ganymede and to characterise the Jupiter system. Planet. Space Sci. 78, 1-21. doi: 10.1016/j.pss.2012.12.002

Gurnett, D. A., Kurth, W. S., Kirchner, D. L., Hospodarsky, G. B., Averkamp, T. F., Zarka, P., et al. (2004). The Cassini radio and plasma wave investigation. Space Sci. Rev. 114, 395-463. doi: 10.1007/s11214-004-1434-0

Gurnett, D. A., Persoon, A. M., Kurth, W. S., Roux, A., and Bolton, S. J. (2000). Plasma densities in the vicinity of Callisto from Galileo plasma wave observations. Geophys. Res. Lett. 27, 1867-1870. doi: 10.1029/2000GL003751

Haider, S. A., Bhardwaj, A., Shanmugam, M., Goyal, S. K., Sheel, V., Pabari, J., et al. (2018). "Indian Mars and Venus missions: science and exploration," in 42nd COSPAR Scientific Assembly. vol. 42 COSPAR Meeting (Pasadena, CA), B4.1-10-18.

Harvey, C. C., Etcheto, J., and Mangeney, A. (1979). Early results from the ISEE electron density experiment. Space Sci. Rev. 23, 39-58. doi: 10.1007/BF00174110

Heikkila, W. J., Smith, J. B., Tarstrup, J., and Winningham, J. D. (1970). The soft particle spectrometer in the ISIS-I satellite. Rev. Sci. Instr. 41, 1393-1402. doi: $10.1063 / 1.1684291$

Huang, C. Y., Burke, W. J., Hardy, D. A., Gough, M. P., James, H. G., Villalón, E., et al. (2001). Electron acceleration by megahertz waves during OEDIPUS C. J. Geophys. Res. 106, 1835-1848. doi: 10.1029/1999JA000286

James, H. G. (1983). Sounder-accelerated particles observed on ISIS. J. Geophys. Res. 88, 4027-4040. doi: 10.1029/JA088iA05p04027

James, H. G. (1987). Discharge of RF-induced spacecraft dc potential by positive ions. Planet. Space Sci. 35, 105-118. doi: 10.1016/0032-0633(87)90149-8

James, H. G., Sotnikov, V. I., Burke, W. J., and Huang, C. Y. (1999). OEDIPUS-C observations of electrons accelerated by radio frequency fields at whistler-mode frequencies. Phys. Plasmas 6, 4058-4069. doi: 10.1063/1.873668

Jia, X., Kivelson, M. G., Khurana, K. K., and Kurth, W. S. (2018). Evidence of a plume on Europa from Galileo magnetic and plasma wave signatures. Nat. Astron. 2, 459-464. doi: 10.1038/s41550-018-0450-Z

Jordan, R., Picardi, G., Plaut, J., Wheeler, K., Kirchner, D., Safaeinili, A., et al. (2009). The Mars express MARSIS sounder instrument. Planet. Space Sci. 57, 1975-1986. doi: 10.1016/j.pss.2009.09.016

Kivelson, M. G., Khurana, K. K., Coroniti, F. V., Joy, S., Russell, C. T., Walker, R. J., et al. (1997). Magnetic field and magnetosphere of Ganymede. Geophys. Res. Lett. 24:2155. doi: 10.1029/97GL02201

Kiwamoto, Y., and Benson, R. F. (1979). Nonlinear Landau damping in the ionosphere. J. Geophys. Res. 84, 4165-4174. doi: 10.1029/JA084iA08p04165
Kliore, A. J., Hinson, D. P., Flasar, F. M., Nagy, A. F., and Cravens, T. E. (1997). The ionosphere of Europa from Galileo radio occultations. Science 277, 355-358. doi: 10.1126/science.277.5324.355

Knecht, R. W., van Zandt, T. E., and Russell, S. (1961). First pulsed radio soundings of the topside of the ionosphere. J. Geophys. Res. 66, 3078-3081. doi: 10.1029/JZ066i009p03078

Lockwood, G. E. K. (1963). Plasma and cyclotron spike phenomena observed in top-side ionograms. Canad. J. Phys. 41:190. doi: 10.1139/ p63-021

McAfee, J. R. (1968). Ray trajectories in an anisotropic plasma near plasma resonance. J. Geophys. Res. 73, 5577-5583. doi: 10.1029/JA073i017p05577

McAfee, J. R. (1969). Topside resonances as oblique echoes. J. Geophys. Res. 74, 802-808. doi: 10.1029/JA074i003p00802

Muldrew, D. B. (1972). Electron resonances observed with topside sounders. Radio Sci. 7, 779-789. doi: 10.1029/RS007i008p00779

Oya, H. (1971). Verification of theory on weak turbulence relating to the sequence of diffuse plasma resonances in space. Phys. Fluids 14, 2487-2499. doi: $10.1063 / 1.1693358$

Paschmann, G., Quinn, J. M., Torbert, R. B., Vaith, H., McIlwain, C. E., Haerendel, G., et al. (2001). The electron drift instrument on cluster: overview of first results. Ann. Geophys. 19, 1273-1288. doi: 10.5194/angeo-191273-2001

Phillips, C. B., and Pappalardo, R. T. (2014). Europa clipper mission concept: exploring Jupiter's ocean moon. EOS Trans. 95, 165-167. doi: 10.1002/2014EO200002

Pulinets, S. A., and Selegei, V. V. (1986). Ionospheric plasma modification in the vicinity of a spacecraft by powerful radio pulses in topside sounding. J. Atmos. Terrest. Phys. 48, 149-157. doi: 10.1016/0021-9169(86)90079-6

Reinisch, B. W., Haines, D. M., Bibl, K., Cheney, G., Galkin, I. A., Huang, X., et al. (2000). The radio plasma imager investigation on the IMAGE spacecraft. Space Sci. Rev. 91, 319-359.

Roth, L., Saur, J., Retherford, K. D., Strobel, D. F., Feldman, P. D., McGrath, M. A., et al. (2014). Transient water vapor at Europa's south pole. Science 343, 171-174. doi: $10.1126 /$ science. 1247051

Russell, C. T. (2005). Interaction of the Galilean moons with their plasma environments. Planet. Space Sci. 53, 473-485. doi: 10.1016/j.pss.2004.05.003

Schroeder, D. M., Romero-Wolf, A., Carrer, L., Grima, C., Campbell, B. A., Kofman, W., et al. (2016). Assessing the potential for passive radio sounding of Europa and Ganymede with RIME and REASON. Planet. Space Sci. 134, 52-60. doi: 10.1016/j.pss.2016.10.007

Serov, A. A., Galperin, I. I., Lisakov, I. V., and Shuiskaia, F. K. (1985). Local acceleration of electrons of a near-satellite plasma by radio emission from a high-power onboard transmitter. Kosmiches. Issledovaniia 23, 431-443.

Shuiskaya, F. K., Galperin, Y. I., Serov, A. A., Baranets, N. V., Kushnerevsky, Y. V., Vasil'ev, G. V., et al. (1990). Resonant heating of the ionospheric plasma by powerful radiopulses aboard the Intercosmos-19 and Cosmos1809 satellites. Planet. Space Sci. 38, 173-180. doi: 10.1016/0032-0633(90) 90081-Z

Stone, R. G., Pedersen, B. M., Harvey, C. C., Canu, P., CornilleauWehrlin, N., Desch, M. D., et al. (1992). ULYSSES radio and plasma wave observations in the Jupiter environment. Science 257, 1524-1531. doi: 10.1126/science.257.5076.1524

Voshchepynets, A., Barabash, S., Ramstad, R., Holmstrom, M., Andrews, D., Frahm, R. A., et al. (2018). Ions accelerated by Sounder-Plasma interaction as observed by Mars express. J. Geophys. Res. 123, 9802-9814. doi: 10.1029/2018JA025889

Conflict of Interest Statement: The authors declare that the research was conducted in the absence of any commercial or financial relationships that could be construed as a potential conflict of interest.

Copyright (@ 2019 Voshchepynets, Barabash, Holmstrom and Frahm. This is an open-access article distributed under the terms of the Creative Commons Attribution License (CC BY). The use, distribution or reproduction in other forums is permitted, provided the original author(s) and the copyright owner(s) are credited and that the original publication in this journal is cited, in accordance with accepted academic practice. No use, distribution or reproduction is permitted which does not comply with these terms. 